\title{
Lymphatic endothelial cell identity is reversible and its maintenance requires Prox1 activity
}

\author{
Nicole C. Johnson, ${ }^{1}$ Miriam E. Dillard, ${ }^{1}$ Peter Baluk, ${ }^{2}$ Donald M. McDonald, ${ }^{2}$ Natasha L. Harvey, ${ }^{3}$ \\ Sharon L. Frase, ${ }^{4}$ Guillermo Oliver ${ }^{1,5}$ \\ ${ }^{1}$ Department of Genetics and Tumor Cell Biology, St. Jude Children's Research Hospital, Memphis, Tennessee 38105, USA; \\ ${ }^{2}$ Cardiovascular Research Institute and Department of Anatomy and Comprehensive Cancer Center, University \\ of California at San Francisco, San Francisco, California 94143, USA; ${ }^{3}$ Division of Hematology, The Hanson Institute, \\ Adelaide, South Australia 5000, Australia; ${ }^{4}$ Electron Microscopy Cell and Tissue Imaging Center, St. Jude Children's \\ Research Hospital, Memphis, Tennessee 38105, USA
}

The activity of the homeobox gene Prox1 is necessary and sufficient for venous blood endothelial cells (BECs) to acquire a lymphatic endothelial cell (LEC) fate. We determined that the differentiated LEC phenotype is a plastic, reprogrammable condition that depends on constant Prox1 activity for its maintenance. We show that conditional down-regulation of Prox1 during embryonic, postnatal, or adult stages is sufficient to reprogram LECs into BECs. Consequently, the identity of the mutant lymphatic vessels is also partially reprogrammed as they acquire some features typical of the blood vasculature. siRNA-mediated down-regulation of Prox1 in LECs in culture demonstrates that reprogramming of LECs into BECs is a Prox1-dependent, cell-autonomous process. We propose that Prox 1 acts as a binary switch that suppresses BEC identity and promotes and maintains LEC identity; switching off Prox1 activity is sufficient to initiate a reprogramming cascade leading to the dedifferentiation of LECs into BECs. Therefore, LECs are one of the few differentiated cell types that require constant expression of a certain gene to maintain their phenotypic identity.

[Keywords: Lymphatic endothelial cells; blood endothelial cells; lymphangiogenesis; reprogramming; Prox1; siRNA]

Supplemental material is available at http://www.genesdev.org.

Received August 12, 2008; revised version accepted October 7, 2008.

During embryonic development, cell type differentiation is considered a unidirectional, irreversible terminal process. However, recent work has challenged these views by showing that individual transcription factors can reprogram differentiated somatic cells (Takahashi et al. 2007), or that mature B cells can be reprogrammed back into functional $\mathrm{T}$ cells by switching off the transcription factor Pax5 (Cobaleda et al. 2007). Developmental plasticity occurs between the pancreas and liver, and ectopic expression of certain transcription factors can promote cell fate reversal in the endoderm (Zaret 2008). In endothelial cells (ECs), arterio-venous identity can be reprogrammed by the disruption or by the forced expression of Notch signaling or the orphan nuclear receptor CoupTFII (You et al. 2005; Roca and Adams 2007), and lymphatic identity can be acquired upon the forced expression of Prox 1 in blood ECs (BECs) maintained in culture (Hong et al. 2002; Petrova et al. 2002).

${ }^{5}$ Corresponding author.

E-MAIL guillermo.oliver@stjude.org; FAX (901) 526-2907.

Article is online at http://www.genesdev.org/cgi/doi/10.1101/gad.1727208.
Despite this evidence for cell plasticity and cell reprogramming, little is known about how cell types maintain their terminally differentiated fate. We demonstrated previously that early during embryonic development, restricted expression of Prox 1 in a subpopulation of venous ECs is the first step in the process leading to lymphatic EC (LEC) specification; Prox $1^{-/-}$embryos lack a lymphatic vasculature and die at around embryonic day 14.5 (E14.5) (Wigle and Oliver 1999). Therefore, we proposed that embryonic venous ECs are the default state, and upon Prox1 expression, they adopt an LEC phenotype (Wigle et al. 2002). Following this specification step, the whole lymphatic vasculature eventually forms in a stepwise process that continues until a few weeks after birth (Oliver and Detmar 2002).

In most genetic backgrounds, Prox $1^{+/-}$pups die shortly after birth and display phenotypes characteristic of lymphatic dysfunction. Those that survive to adulthood display a less severe lymphatic phenotype; their lymphatic vasculature is mispatterned and leaky (Harvey et al. 2005). These results argue that lymphatic vasculature formation is highly susceptible to Proxl dosage. In addi- 
tion, Prox 1 expression in LECs is constantly maintained during embryonic and postnatal stages (our unpublished results). Thus, we questioned: which aspects of developmental and postnatal lymphangiogenesis require Prox1 function? To address this question we conditionally removed Prox 1 activity in a time-specific manner by using a ubiquitously expressed inducible Cre mouse strain.

Using this approach we show that conditional downregulation of Proxl is sufficient to initiate a reprogramming cascade that dedifferentiates LECs into BECs, and consequently, the identity of lymphatic vessels is also partially reprogrammed. Furthermore, siRNA-mediated down-regulation of Prox 1 in LECs in culture demonstrates that the reprogramming of LECs into BECs is a Prox1-dependent, cell-autonomous process.

These results demonstrate that the LEC phenotype is a labile, reversible condition that requires constant levels of Prox1 expression for its maintenance. We propose that Prox1 acts as a key binary switch necessary to suppress BEC identity and promote and maintain LEC fate. Alterations in the levels of Proxl expression are sufficient to dedifferentiate LECs into BECs. Therefore, this is one of the few examples of a gene (Prox1) whose activity is required not only for cell type specification (LEC identity) but also to maintain the mature differentiated LEC fate.

\section{Results}

Time-specific deletion of Proxl results in blood-filled vessels

To conditionally remove Prox1 activity in a time-specific manner we used the ubiquitously expressed tamoxifen minumum (TM)-inducible mouse strain CAGGCre$E R^{T M}$ (Hayashi and McMahon 2002). CAGGCre$E R^{T M}$;Prox $1^{10 x P /+}$ and Prox $1^{\text {loxP/loxP }}$ mice (Harvey et al. 2005) were intercrossed, and pregnant dams were initially injected either with $1 \mathrm{mg}$ per day TM at E8.5, E9.5, and E10.5 (to remove Prox 1 from venous LEC precursors) or with $3 \mathrm{mg}$ per day at E12.5 and E13.5 (to remove Prox1 from LECs in the forming lymph sacs). TM dosage was determined based on survival and optimal efficiency of Prox1 deletion. In both cases, embryos were isolated at E14.5.

Visual inspection of the isolated embryos showed normal-looking CAGGCre-ER ${ }^{T M}$;Prox $1^{\text {loxP/loxP }}$ embryos when TM was not administered (Fig. 1A). Instead, dermal edema (Fig. 1B, arrows) and scattered blood-filled vessels (Fig. 1B, arrowhead) were detected in TM-treated embryos in which Prox 1 was excised from venous LEC precursors at early developmental stages. A much larger number of scattered, superficial, mispatterned, and blood-filled vessels were observed in conditional mutant embryos in which Prox 1 was excised from developing lymph sacs at later stages (Fig. 1C, arrowheads). The distribution of these blood-filled vessels was reminiscent of the developing superficial lymphatic vessels originally described by Sabin (1902).

Next, Prox1 activity was deleted after the initial lymphatic network was formed ( $3 \mathrm{mg}$ of TM was injected at
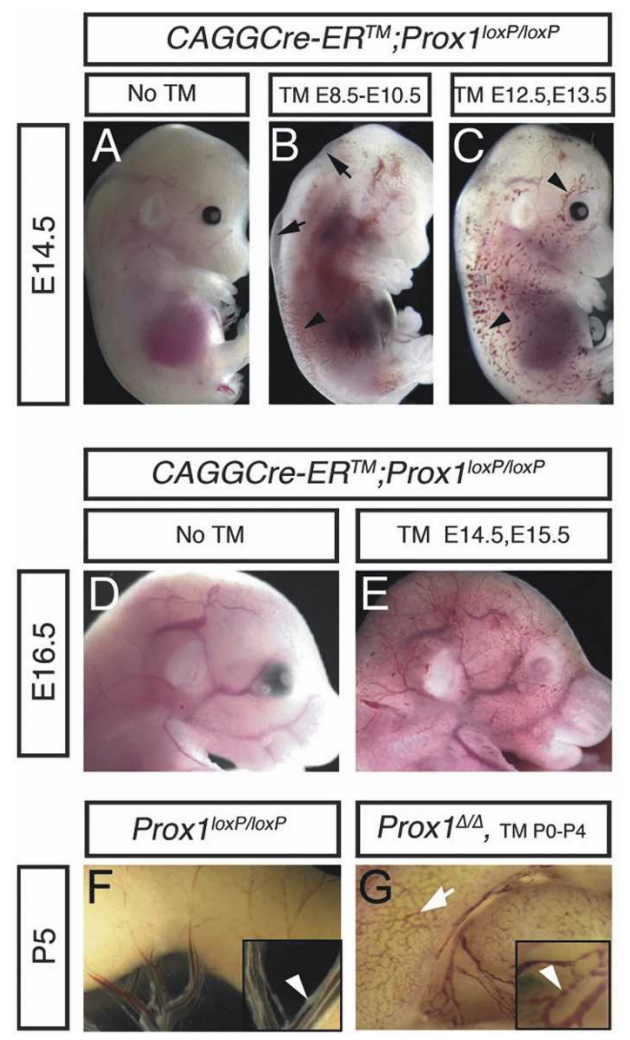

Figure 1. Conditional deletion of Prox 1 during embryogenesis and postnatal stages results in blood-filled dermal and mesenteric lymphatics. (A) Control E14.5 CAGGCre-ER ${ }^{T M}$;Prox $1^{\text {loxP/loxP }}$ embryo without TM induction. (B) E14.5 CAGGCre$E R^{T M}$;Prox $1^{\text {1oxP/loxP }}$ embryo exposed to TM at E8.5, E9.5, and E10.5 exhibit edema (arrows) and some blood-filled dermal vessels (arrowhead). (C) TM exposure at E12.5 and E13.5 resulted in a much larger number of blood-filled dermal vessels (arrowheads). (D) Control E16.5 CAGGCre-ER ${ }^{T M}$;Prox1 1oxP/loxP $\mathrm{em}$ bryo without TM induction. $(E)$ Blood-filled dermal vessels were also observed in E16.5 CAGGCre-ER ${ }^{T M}$;Prox $1^{\text {loxP/loxP }}$ embryos after Prox1 deletion at E14.5 and E15.5. Control Prox $1^{\operatorname{lox} P / \operatorname{lox} P}(F)$ and CAGGERCre-ER ${ }^{T M}$ Prox $1^{\text {loxP/loxP }}\left(\right.$ Prox $\left.^{\Delta / \Delta}\right)(G)$ P0 pups were fed with TM from P0 to P5 and examined at P5. $(G)$ Blood was seen in the submucosal lymphatics (arrow) of Prox $1^{\Delta / \Delta}$ pups and in all vessels of the mesenteric membrane (inset, arrowhead), but not in littermate controls where normal-looking lymphatics and valves (arrowhead) are seen (as shown in $F$ ).

E14.5 and E15.5), and CAGGCre-ER ${ }^{T M} ; \operatorname{Prox}^{10 x P / \operatorname{lox} P}$ conditional-mutant embryos were analyzed at E16.5. As expected, normal-looking embryos were observed in the absence of TM (Fig. 1D); however, an extended network of blood-filled superficial vessels (also reminiscent of superficial lymphatic vessels) was present in the conditional mutant embryos at this stage (Fig. 1E).

Lymphatic vasculature remodeling and maturation continues for at least $2 \mathrm{wk}$ after birth (Karpanen et al. 2006), and Proxl is expressed in LECs throughout life. Therefore, we orally administered $20 \mu \mathrm{g}$ of TM per day to newborn CAGGCre-ER ${ }^{T M}$;Prox $1^{\operatorname{lox} P / \text { loxP }}$ pups, starting at postnatal day $0(\mathrm{P0})$ until P5 (most conditional mutant pups did not survive beyond P6). Detailed examination 
of the intestinal lymphatic vasculature of these conditional Prox1 mutant pups revealed the presence of blood in most of the submucosal (Fig. 1G, arrow) and mesenteric lymphatics (Fig. 1G, inset, arrowhead). The fact that Prox 1 activity is not involved in the development, function, or maintenance of blood vessels argues that the blood-filled vasculature observed in the conditional mutant embryos at various developmental stages corresponded to defective lymphatic vessels. Additional macroscopic and confocal imaging performed with other tissues isolated from the conditional mutants at postnatal stages revealed the widespread characteristic of the blood-filled lymphatic phenotype and that the blood was not from hemorrhage; rather, it was inside thin-walled vessels (Fig. 2). Together, these results suggest that, although with different severity, conditional inactivation of Prox 1 at any stage of embryonic or postnatal lymphangiogenesis results in mispatterned and blood-filled lymphatic vessels.

\section{Conditional deletion of Proxl dedifferentiates LECs into BECs}

Next, the identity of the blood-filled vessels was examined at the molecular level by labeling transverse sections of conditional mutant embryos and pups with a combination of antibodies for LECs and BECs. Very few lymphatic vessels were identified in mutant embryos in which TM administration was initiated early during development (E8.5) (data not shown). As expected, the lymphatic vasculature of control Prox $1^{\operatorname{lox} P / \operatorname{lox} P}$ embryos and pups expressed typical LEC markers such as Prox1, secondary lymphocyte chemokine (Slc) (Kriehuber et al. 2001), and Podoplanin (Pdpn) (Fig. 3A,B,E,F,I,J; Schacht et al. 2003) and were devoid of BEC markers such as Endoglin (Eng) (Wikstrom et al. 2002) and Cd34 (Fig. 3C,D,G,H,K,L; Kriehuber et al. 2001). In the conditional mutants and as expected for Cre-mediated recombination, the extent of Prox1 deletion was variable (Fig. $3 \mathrm{~A}^{\prime}, \mathrm{B}^{\prime}, \mathrm{E}^{\prime}, \mathrm{F}^{\prime}, \mathrm{I}^{\prime}, \mathrm{J}^{\prime}$; Supplemental Fig. 1). Despite this variability, Prox1 expression was significantly reduced in most of the conditional mutant lymphatic vessels, and in many cells, it was lost altogether (Fig. 3). In tight association with the down-regulation of the expression of Prox1, that of Slc and Pdpn was not detected (Fig. $\left.3 \mathrm{~A}^{\prime}, \mathrm{B}^{\prime}, \mathrm{E}^{\prime}, \mathrm{F}^{\prime}, \mathrm{I}^{\prime}, \mathrm{J}^{\prime}\right)$. Lyve1 (protein or mRNA) was the only LEC marker analyzed that was not completely suppressed in the mutant lymphatic vessels, although its expression was greatly reduced and almost extinguished in some mutant vessels (Figs. 2B,D, 5D; data not shown). Interestingly, the reduction of Proxl expression below a certain threshold was sufficient to suppress the expres-

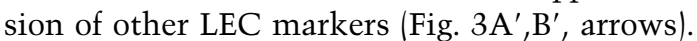

Concomitant with the down-regulation of LEC markers, conditional mutant vessels ectopically expressed BEC-specific markers such as Eng and Cd34 (Fig. $\left.3 \mathrm{C}^{\prime}, \mathrm{D}^{\prime}, \mathrm{G}^{\prime}, \mathrm{H}^{\prime}, \mathrm{K}^{\prime}, \mathrm{L}^{\prime}\right)$. To determine whether similar results could also be obtained upon Prox 1 inactivation in adult lymphatic vessels, we administered $1 \mathrm{mg}$ TM weekly between 4 and 8 mo of age to CAGGCre-
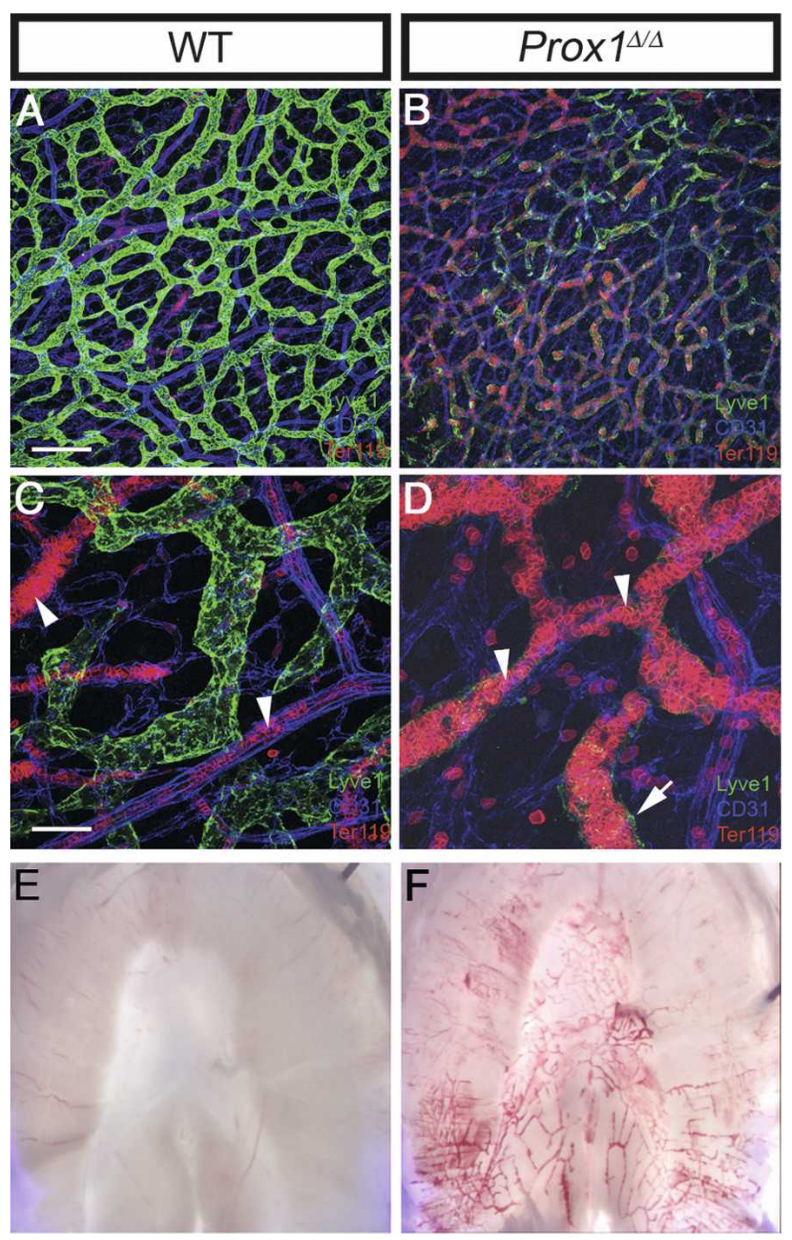

Figure 2. Mutant vessels are blood filled. $(A-D)$ Whole-mount preparations of mouse neonatal intestine immunostained with markers for lymphatics (Lyve1, green), erythrocytes (TER119, red), and endothelial junctions (CD31, blue). Wild-type mice $(A, C)$ and conditional Prox 1 -mutant mice $(B, D)$ were treated from P0 to P5 with TM to delete Prox1. The intensity of Lyve1 expression was greatly reduced $(B)$ or almost absent $(D$, arrow $)$ in the mesenteric and submucosal lymphatics of the conditional mutant vessels. Erythrocytes were confined to blood vessels in the wild-type mice $(C$, arrowheads), but were found within the mutant lymphatics of $\operatorname{Prox}^{\Delta / \Delta}$ littermates (D, arrowheads). $C$ and $D$ are enlargements of regions of $A$ and $B$. (E) Bright-field microscopy of P5 wild-type diaphragm compared with that of the Prox $1^{\Delta / \Delta}$ littermate $(F)$ showing blood-filled lymphatics. Bars: $A, B, 200 \mu \mathrm{m} ; C, D, 50 \mu \mathrm{m}$.

$E R^{T M}$;Prox $1^{\operatorname{lox} P / \operatorname{lox} P}$ mice. Most likely because the adult lymphatic vasculature is mostly quiescent and therefore the chromatin will be tightly packed, the extent of Prox 1 down-regulation in these mice was very inefficient; however, analysis of their dermal lymphatics revealed that, similar to deletion at earlier stages, Prox1 excision during later postnatal stages was accompanied by the downregulation of LEC markers and ectopic expression of BEC markers in affected conditional mutant vessels (Fig. $\left.3 \mathrm{M}^{\prime}-\mathrm{P}^{\prime}\right)$.

We showed previously that Prox1 gene dosage is crucial for postnatal survival; i.e., in most genetic back- 

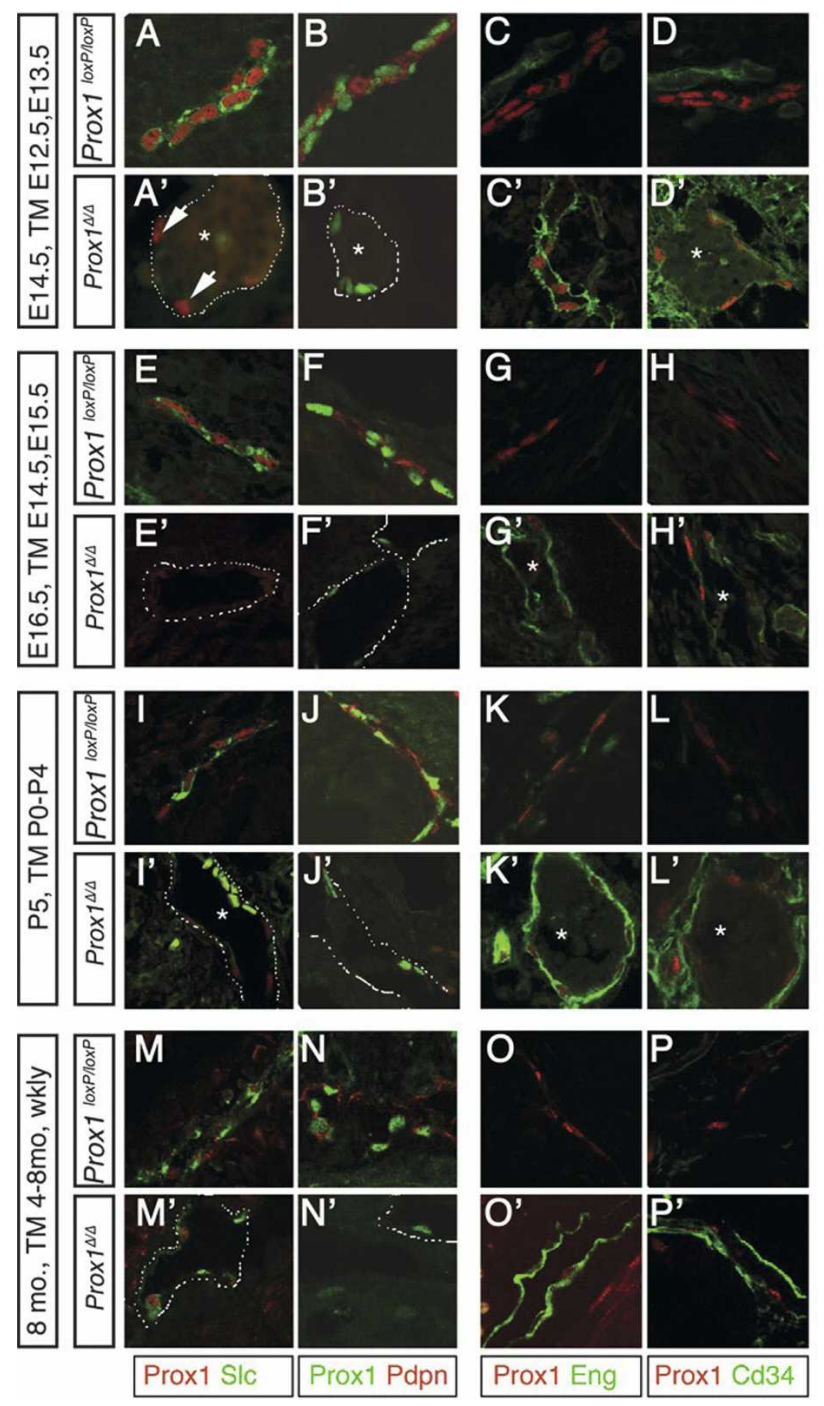

Figure 3. Prox 1 expression is constantly required for the maintenance of LEC fate. Prox1 deletion at embryonic $\left(A^{\prime}, B^{\prime}\right.$, $\left.C^{\prime}, D^{\prime}, E^{\prime}, F^{\prime}, G^{\prime}, H^{\prime}\right)$ or postnatal $\left(I^{\prime}, J^{\prime}, K^{\prime}, L^{\prime}, M^{\prime}, N^{\prime}, O^{\prime}, P^{\prime}\right)$ stages resulted in the loss of LEC fate and gain of BEC identity. Sections from the thoracic cavity and skin of control Prox $1^{10 x P / / 0 x P}$ and Prox $^{\Delta / \Delta}$ embryos at E14.5 (with TM-mediated Prox1 deletion at E12.5 and E13.5), E16.5 (with Prox1 deletion at E14.5 and E15.5), and from submucosal lymphatics of P5 pups (with Prox1 deletion from P0-P5) and skin from 8-mo-old mice (with Prox1 deletion from 4-8 mol were immunostained using antibodies specific for LECs and BECs. Wild-type lymphatics expressed the LEC markers Slc and Pdpn, but they did not express the BEC markers Eng and Cd34. Expression of the LEC markers was drastically reduced or absent in Prox $1^{\Delta / \Delta}$ littermates, and BEC markers were ectopically expressed. Arrows in $A^{\prime}$ highlight that partial reduction of Prox 1 expression levels is sufficient to abolish the expression of the analyzed LEC markers. Asterisks indicate the presence of blood inside the conditional mutant vessels. Dashed lines labels the mutant lymphatic vessel.

grounds, Prox $1^{+/-}$pups die shortly after birth and display phenotypes characteristic of lymphatic dysfunction (i.e., chyle accumulation in the thoracic cavity and sometimes in the peritoneal cavity) (Wigle and Oliver 1999;
Harvey et al. 2005). Analysis of the severely affected lymphatic vasculature of these nonsurviving Prox $1^{+/-}$ newborn pups revealed features similar to those observed in the conditional mutant embryos and pups. As shown in Figure 4B, the submucosal and mesenteric lymphatics of nonsurviving P2 Prox $1^{+/-}$pups were mispatterned and predominantly blood filled. In addition, the identity of some vessels was affected; i.e., they suppressed the expression of LEC markers and ectopically expressed BEC markers (Fig. 4).

Proxl mutant vessels exhibit disrupted cell adhesion properties and display ectopic pericyte investment

Next we investigated whether the switch from LEC fate toward BEC fate was accompanied by a switch in vessel characteristics; from a lymphatic vessel toward a blood vessel.

Vascular endothelial (VE)-cadherin is an endothelialspecific adhesion molecule located at junctions between

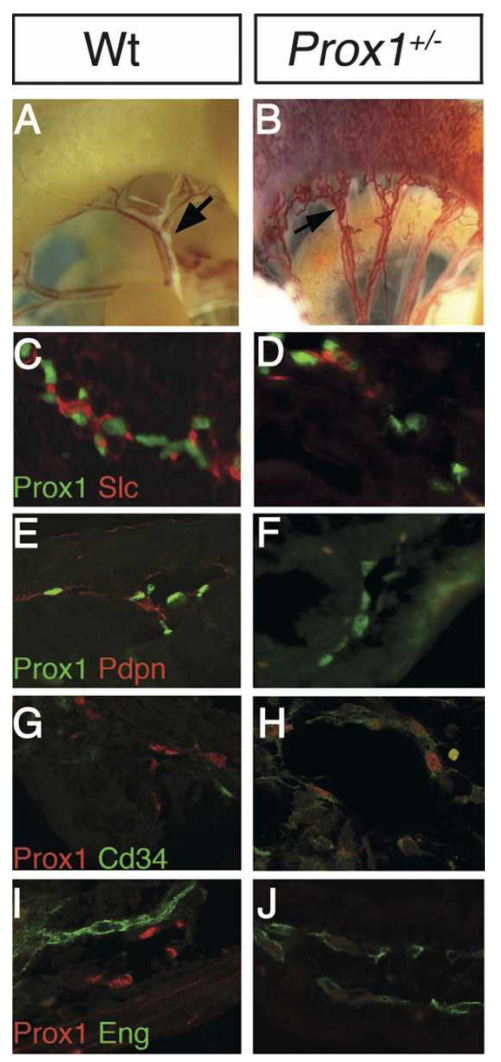

Figure 4. Nonsurviving P0 Prox $1^{+/-}$pups display aberrant LECand BEC-specific markers expression. $(A)$ Bright-field microscopy of a P0 wild-type pup revealed the normal lymphatic architecture of the intestine mesenteric membrane and the lymphatic valves (arrow). (B) In nonsurviving Prox $1^{+/-}$littermates, submucosal and mesenteric vessels were abnormally blood filled. In the submucosal lymphatics of immunostained sections from the Prox $1^{+-}$pups, the expression of LEC markers was reduced or absent $(D, F)$, and ectopic expression of the BEC markers $\mathrm{Cd} 34$ and Eng was observed $(H, J)$. 
ECs (Breier et al. 1996). VE-cadherin antibodies have helped identify the differences in the way that LECs from initial (discontinuous buttons) or collecting (continuous zippers) lymphatics of the mouse trachea are joined (Baluk et al. 2007). We took advantage of this feature to determine whether the switch from LEC fate toward BEC fate observed in the mutant vessels was accompanied by alterations in some of the cell adhesion properties characteristic of lymphatic vessels.

Typical VE-cadherin-stained button-like junctions were seen in the Lyve $1^{+}$lymphatics of P5 wild-type intestinal lymphatics (Fig. 5A [arrows], C [arrows]). In contrast, VE-cadherin staining was reduced, disrupted, or absent in the conditional mutant vessels in which Lyve1 expression was also down-regulated (Fig. 5B [arrow], D). Electron microscopic analysis of Lyve1-immunostained mesenteric lymphatics (Lyvel signal was enhanced by the use of diaminobenzidine as a substrate) from a P5 wild-type pup showed normal pericyte-free initial lymphatics (Fig. 6A). Instead, many of the blood-filled lymphatics of P5 Prox1 conditional mutant pups (not shown) and those of nonsurviving Prox $1^{+/-}$pups, were abnormally surrounded by pericyte-like cells (Fig. 6B,C, red arrowheads). The pericyte identity was confirmed upon immunohistochemical analysis of the mutant vessels with the pericyte markers smooth muscle actin (SMA), chondroitin sulfate proteoglycan 4 (Cspg4/NG2), and Pdgfrß (Fig. 6D-K; Lindahl et al. 1997; Ozerdem et al. 2002; Makinen et al. 2007). In addition, we determined that these blood-filled mutant vessels also abnormally express platelet markers such as fibrin and CD41 (Supplemental Fig. 2). Together, these results indicate that down-regulation of Proxl expression not only dedifferentiates LEC fate toward BEC, but also and as a consequence, it promotes the loss of typical lymphatic cell junction characteristics (VE-cadherin) and the gain of blood vessel features (pericytes and platelets). This result is significant because it argues that a change in LEC fate is accompanied by at least a partial change in vascular identity.

\section{Prox1 conditional mutant pups display aberrant connections between the lymphatic and blood vasculatures}

What causes the abnormal blood-filled phenotype observed in the Prox1 mutant vessels? Blood-filled lymphatics have been reported in some mouse mutant models; e.g., Slp-76, Syk (Abtahian et al. 2003), Fiaf (Backhed et al. 2007), ephrinB2 (Makinen et al. 2005), and Foxc2 (Petrova et al. 2004). However, the precise cause of these blood-filled lymphatic phenotypes is not yet known. In Syk mutant mice and S1p76 mutant mice, an abnormal, persistent connection between the blood vasculature and the forming lymph sacs has been proposed as the cause of the phenotype (Abtahian et al. 2003). However, in Prox $^{+/-}$mice, the blood-filled lymphatic phenotype is not caused by an abnormal connection between the blood vasculature and the forming lymph sacs (data not shown).
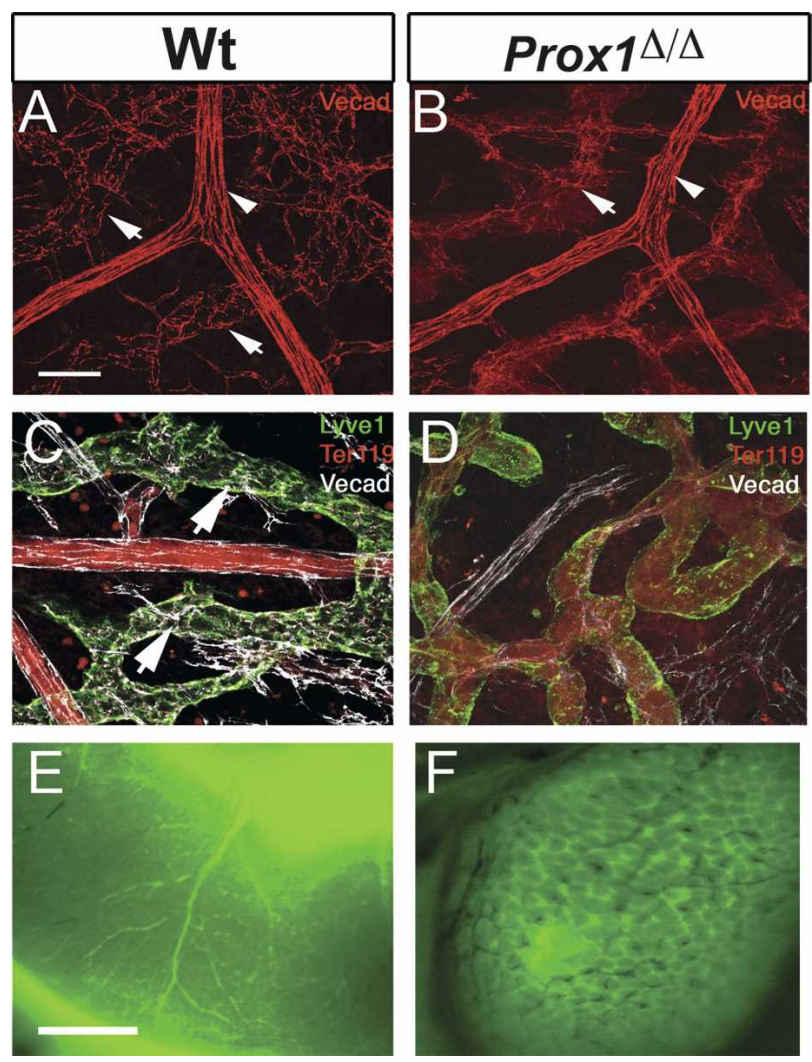

Figure 5. Prox1 mutant vessels exhibit disrupted cell adhesion properties and aberrant connections between the lymphatic vasculature and blood vasculature. (A) VE-cadherin expression was found in button-like junctions between LECs of submucosal lymphatics of P5 wild-type intestines (arrows). Strong uniform expression was seen in blood vessels (arrowhead). (B) In contrast, VE-cadherin staining was weak or absent in the lymphatics of conditional mutant littermates (arrow). (C) VE-cadherin (white) expression was found in normal button-like junctions between the LECs of the Lyve $1^{+}$(green) submucosal lymphatics of P5 wild-type intestines (arrows). ( $D$ ) In contrast, VE-cadherin staining was weak or absent in the lymphatics of conditional mutant littermates, where Lyve1 expression level was downregulated. Ter119 (red) staining within the conditional mutant lymphatic also indicated the presence of red blood cells. $(E)$ Cardiac perfusion of FITC-dextran labeled the blood vasculature of the P5 wild-type intestine. (F) A similar assay performed on P5 Prox $1^{\Delta / \Delta}$ littermates labeled not only the blood vasculature but also all of the submucosal lymphatics. The black small vessels seen in this panel correspond to blood-filled small capillaries that were not perfused with the PFA/FITC-dextran solution. Bars: $A, B, 50 \mu \mathrm{m} ; E, F, 1 \mathrm{~mm}$.

Changes in the arterio-venous expression profile result in vascular malformations such as arteriovenous shunts (Roca and Adams 2007). In Prox1 conditional mutants the switch in LEC fate was accompanied by alterations in the expression of adhesion molecules (VE-cadherin), and by the acquisition of some blood vessel-specific features. To investigate whether the chimeric mutant vessels could have established abnormal anastomoses with nearby blood vessels and capillaries, we perfused (via intracardiac injection) FITC-dextran 

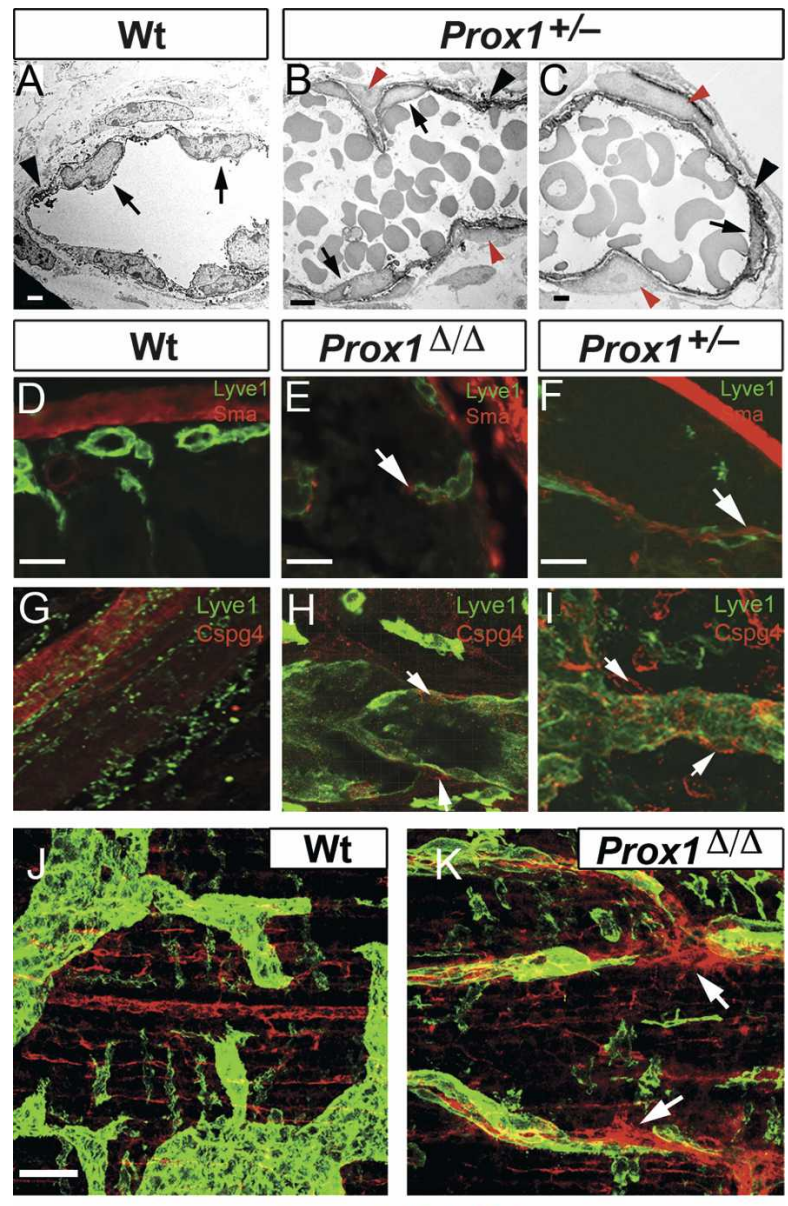

\section{Lyve1/Pdgfr $\beta$}

Figure 6. Prox1 mutant pups display ectopic pericyte investment. (A) Electron microscopic imaging of DAB Lyve1-stained (arrowhead) wild-type submucosal lymphatics exhibiting their typical morphology, ECs are indicated by the arrows. Images from Prox $^{+/-}(B, C)$ pups revealed the abnormal presence of Lyve1 pericytes (red arrowheads) around some mutant lymphatic vessels. Wild-type lymphatics were devoid of $\mathrm{Sma}^{+}(D)$, $\operatorname{Cspg}^{+}(G)$, and Pdgfr $\beta^{+}(J)$ pericytes. $(E, F, H, I, K)$ Abnormal ectopic expression of Sma, Cspg4, and PDGFR $\beta$ (arrows) was detected in some of the lymphatics of the conditional mutant and Prox $1^{+/-}$pups. Bars: $A, 4 \mu \mathrm{m} ; B, 8 \mu \mathrm{m} ; C, 3 \mu \mathrm{m} ; D-I, 50 \mu \mathrm{m} ; I, K$, $50 \mu \mathrm{m}$.

through P5 wild-type and Prox1 conditional mutant pups. FITC-dextran perfused only through the blood vessels of wild-type intestines of P5 pups (Fig. 5E); however, it perfused throughout the whole honeycomb-like submucosal network of lymphatic vessels in the intestines of some conditional mutant pups (Fig. 5F, arrow). Thus, blood abnormally enters the mutant lymphatics of these conditional mutant neonates.

\section{Knockdown of PROX1 expression in cultured LECs is sufficient to reprogram LECs into BECs}

Prox1 activity is necessary and sufficient to convert BECs into LECs in vivo (Wigle et al. 2002) and in vitro
(Hong et al. 2002; Petrova et al. 2002). Here we demonstrated that below a certain threshold of Proxl activity, the LEC phenotype is lost, and mutant cells revert toward a BEC fate. Therefore, we tested whether knockdown of PROX1 via siRNAs in primary cultures of human LECs (hLECs) is sufficient to dedifferentiate these cells into BECs. Following transfection with siRNA against PROX1 or a scrambled control siRNA, hLECs were grown on coverslips for $72 \mathrm{~h}$ and immunostained using the same LEC- and BEC-specific markers used in the mouse studies.

As expected, we detected no changes in PROX1 expression in the hLECs transfected with the scrambled siRNA (Fig. 7A,C,E,G); hLECs transfected with the $S M A R T$ pool siPROX1 reagent showed varied responses; i.e., PROX1 expression was reduced in some cells and absent in others (Fig. 7). The hLECs with reduced PROX1 expression expressed little or no SLC (Fig. 7B) or PDPN (Fig. 7D). The expression of FOXC2 (Petrova et al. 2004) and that of $\beta$-TUBULIN (Kang and Lee 2006), another marker expressed primarily in lymphatic valves, were also down-regulated (data not shown). Conversely, the hLECs ectopically expressed the BEC markers ENG (Fig. 7F) and CD34 (Fig. 7H). Therefore, PROX1 activity is cell-autonomously required to maintain hLEC identity in vitro.

\section{Discussion}

The classic belief that during differentiation irreversible cell fates are established and maintained has recently
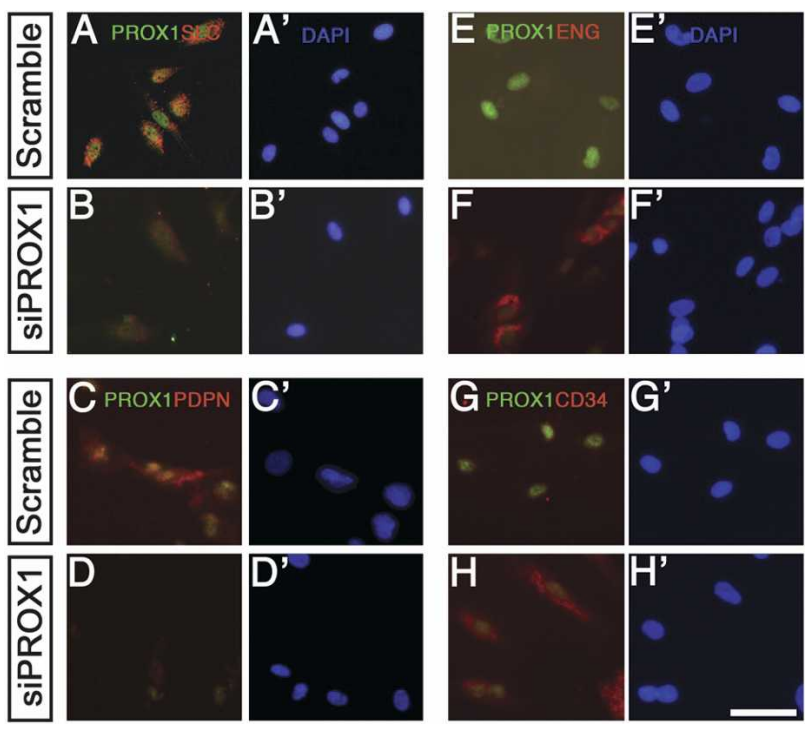

Figure 7. siRNA knockdown of PROX1 expression in LECs in vitro is sufficient to dedifferentiate LECs into BECs. Primary human LECs were maintained in culture and treated with either siProx1 $(B, D, F, H)$ or scrambled siRNA $(A, C, E, G)$. After $72 \mathrm{~h}$, cells were immunostained, and their expression profiles were analyzed. In most cases, PROX1 was effectively down-regulated, and this decrease was directly associated with the loss of the LEC markers SLC $(B)$ and PDPN $(D)$ and the ectopic gain of BEC markers ENG $(F)$ and CD34 $(H) .\left(A^{\prime}-H^{\prime}\right)$ All cells were counterstained with DAPI. Bar, $25 \mu \mathrm{m}$. 
been challenged (Cobaleda et al. 2007; Takahashi et al. 2007). For example, a recent study has shown that conditional inactivation of $P a \times 5$ in $\mathrm{B}$ cells is sufficient to dedifferentiate those cells back into functional $\mathrm{T}$ cells (Cobaleda et al. 2007), and another has shown that forced expression of the transcription factor C/EBP in cultured B lymphocytes represses Pax5 expression, thereby leading to the transformation of these cells into macrophages (Xie et al. 2004). These results highlight the plasticity of lymphocytes (Cobaleda and Busslinger 2008).

Other work has suggested that during differentiation certain cell fates are superimposed on pre-existing ones. In this context, transcription factors could repress a preexisting cell fate and/or activate a new fate. For example, maintaining venous identity requires that the transcription factor COUP-TFII repress Notch signaling in veins, thereby suppressing the expression of arterial-specific genes (You et al. 2005). COUP-TFII gain of function in the endothelium down-regulates the expression of arterial markers, while the loss of COUP-TFII function in venous cells derepresses the arterial program and converts veins into arteries (You et al. 2005).

We determined that the phenotype of embryonic and postnatal LECs is extraordinarily plastic, and that constant levels of Prox1 expression are required throughout the life of LECs to maintain their differentiated identity. We showed that reducing the levels of Prox1 during embryonic and early postnatal lymphangiogenesis is sufficient to initiate a reprogramming cascade leading to the dedifferentiation (reprogramming) of LECs into BECs. Surprisingly, this cell fate conversion is also sufficient to at least partially reprogram the mutant lymphatic vessels into chimeric vessels that have certain blood vasculature characteristics and transport blood. Whether the blood-filled lymphatic phenotype is uniquely caused by ectopic connections established between the chimeric lymphatic vasculature and the surrounding blood vasculature, or could also be influenced by the defective mutant lymphatic vasculature is not yet known. We also do not know whether aberrant ectopic connections between the chimeric lymphatics and the surrounding blood vasculature will form due to this mixed cell identity or because of a direct role of Prox1. This chimeric identity may disrupt signaling pathways that regulate normal lympho-venous separation, thereby resulting in the establishment of ectopic connections. Furthermore, the change in expression of cell adhesion molecules and/or the abnormal presence of pericytes could also contribute to the establishment of abnormal vessel anastomoses and, as a consequence, the blood-filled lymphatic phenotype. Ectopic pericyte coverage by lymphatic capillaries and abnormal accumulation of blood in mesenteric lymphatics has been reported in mutant mice with a mural cell-specific inactivation of Ephrin B2 (Foo et al. 2006).

Early during embryogenesis, Prox 1 is necessary and sufficient to specify the LEC phenotype in venous LEC progenitors (Wigle et al. 2002). We now show that constant levels of Proxl activity in the embryonic and mature lymphatic vasculature are crucial to repress the default BEC program and maintain LEC fate. Thus, we pro- pose that Prox1 acts as a binary switch /Oliver and Srinivasan 2008) whose activity is constantly required to suppress the default BEC identity and promote and maintain that of LECs during differentiation and adulthood. It could be argued that the identity of any of the three main types of ECs (arterial, venous, and lymphatic) can be superimposed on either of the other types by subtle alterations in the combination or level of gene expression. As mentioned above, abnormal pericyte investment and a variable blood-filled lymphatic phenotype have been described previously in some mouse models (Abtahian et al. 2003; Petrova et al. 2004; Foo et al. 2006; Backhed et al. 2007). Whether these phenotypes are directly related to the down-regulation of Proxl in LECs requires further investigation.

Why is Prox 1 expression in LECs constantly required? In general, the specification of the EC phenotype appears to be very labile. For example, it can be influenced by tumor environment (Dudley et al. 2008; Verfaillie 2008). In fact, recent work has demonstrated that ECs derived from mouse prostate tumors and maintained in culture can differentiate into blood vessels or, surprisingly, into cells with osteogenic and chondrogenic features (Dudley et al. 2008).

Therefore, we can speculate that the constant expression of Prox 1 in LECs helps to maintain their differentiated phenotype, and under normal conditions, these cells remain as LECs independent of the environment. However, in a pathologic setting (e.g., inflammation, tumorigenesis), an abnormal environment (e.g., tumor cells) could alter the normal levels of Proxl expression and ultimately promote the dedifferentiation of LECs into BECs.

Lineage switches are observed in various human malignancies. In angiosarcomas, intratumoral capillaries express a mixed blood/lymphatic phenotype (BreitenederGeleff et al. 1999), and highly proliferative lymphatic vessels within tumors express CD34, a BEC surface antigen not expressed by normal adult lymphatics (Fiedler et al. 2006). In Kaposi sarcoma, BEC to LEC switches have been reported and spindle cells have been shown to express LEC and BEC markers (Hong et al. 2004; Wang et al. 2004).

A study of newly formed vascular networks in tumors reported blood vessels with structural heterogeneity and dysfunction (Abramsson et al. 2003). As shown in Supplemental Figure 3, blood-filled vessels with a chimeric blood/lymphatic identity were also found in B-cell

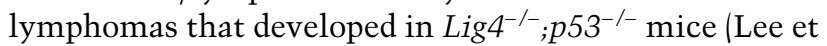
al. 2003). Therefore, it could be argued that the chimeric vessel phenotype resulting from the loss of Proxl mimics the lymphatic chimerism observed in some of these pathologic conditions. Furthermore, this chimerism can cause abnormal anastomoses of neighboring blood and chimeric mutant lymphatic vessels. In tumors, this vascular chimerism and anastomoses could facilitate growth, angiogenesis, and metastasis. By altering their identity or connecting with growing vessels, tumor cells may bypass normal growth restrictions. The identified plasticity of differentiated LECs might help explain lin- 
eage switches reported in some types of malignancies. We speculate that a reversal of the LEC identity via down-regulation of Prox1 levels is one mechanism that operates during some of these pathologic conditions.

Another interesting question is why mature LECs retain the ability to dedifferentiate? Currently, and based on the available data we can only speculate, but it could be argued that EC plasticity might allow the vasculature to rapidly respond to physiological challenges, as reflected, for example, by data showing that hemodynamics can influence/reprogram arterial and venous EC identity (Jones et al. 2006). Interestingly, recent data on the Slp76 mutant mice revealed that in the blood-filled lymphatics, blood flow negatively regulates LEC fate by down-regulating Prox1 expression (C.-Y. Chen, C.C. Bertozzi, L. Yuan, J.S. Lee, M.M. Lu, S.J. Stachelek, P. Mericko, R.J. Levy, and M.L. Kahn, in prep.). Furthermore, it could also be argued that in certain conditions requiring a rapid additional supply of blood (a unique angiogenesis-promoted formation of new vessels might not be quick enough), the formation of new vessels solely via an angiogenic mechanism may not satisfy tissue metabolic requirements quickly enough. Perhaps then, dedifferentiation of a nearby lymphatic network (down-regulation of Prox1 would be the triggering event) into blood vessels could provide an additional, more rapid source of blood.

A better knowledge of cellular plasticity, reprogramming, and gene modulation should provide us with a better understanding of EC identity and could facilitate the conversion of one cell type into another.

In summary, our results demonstrate that LEC fate is plastic. The identity of the three main types of ECs (arterial, venous, and lymphatic) can be superimposed or reverted by subtle alterations in the combination or levels of expression of a few master regulators (e.g., Notch signaling, Coup-TFII, Prox1). This change in cell fate appears to be sufficient to promote alterations in vessel identity and function, and this plasticity could be extremely versatile during normal and pathologic vessel growth.

\section{Materials and methods}

\section{Mouse experiments}

All experiments using animals were approved by and conducted in accordance with St. Jude Children's Research Hospital Animal Care and Use Committee guidelines. Prox $1^{+/-}$mice and Prox $1^{-/-}$embryos were generated as reported previously (Wigle and Oliver 1999). The conditional $\operatorname{Prox} 1^{\operatorname{lox} P}$ mouse strain was previously reported (Harvey et al. 2005). The CAGGCre-ER ${ }^{T M}$ strain was provided by Dr. A. McMahon (Hayashi and McMahon 2002). For the inducible Proxl excision experiments, TM (Sigma) was dissolved in safflower oil $(20 \mathrm{mg} / \mathrm{mL})$ and injected intraperitoneally into pregnant dams daily starting at E8.5 $(1 \mathrm{mg}$ per day for $3 \mathrm{~d}$ ) or starting at E12.5 (3 mg per day for $2 \mathrm{~d}$ ). Embryos were collected at E14.5. For late embryonic Prox1 excision, pregnant dams were injected at E14.5 (5 mg per day for 2 d), and embryos were collected at E16.5. Perinatal Prox1 excision was performed by daily oral administration of $20 \mu \mathrm{g}$ of TM per day to CAGGCre-ER;Prox $1^{10 x P / l o x P}$ neonates and wild-type littermates for five consecutive days (P0-P5). Adult excision experiments involved injecting CAGGCre-ER ${ }^{T M}$;Prox $1^{10 x P / / 0 x P}$ mice with $1 \mathrm{mg}$ of TM weekly from 4 to 8 mo of age. Prox $1^{\text {loxp } / \text { lox } P}$ littermates injected with TM on the same schedule were used as a control. Proxl excision was quantified by examining at least three lymphatic vessels in at least 10 consecutive $10-\mu \mathrm{m}$ sections. Lyve $1^{+}$, Prox $1^{+}$, dapi ${ }^{+}$cells were counted and reported as a percentage of $\mathrm{Lyve}^{+}{ }^{+}$, dapi ${ }^{+}$cells in the vessel. Statistical data was generated using the Student's $t$-test. Tumor samples were obtained from Lig4 ${ }^{-/-} ; p 53^{-/-}$mice (Lee et al. 2003) kindly provided by Drs. Y. Lee and P. McKinnon.

\section{Immunohistochemical analysis}

The intestines were dissected from P0 Prox $1^{+/-}$pups and P5 $C A G G C r e-E R ; \operatorname{PrOx} 1^{10 \times P / / 0 \times P}$ pups and their corresponding wildtype littermates. For immunohistochemical analysis of the submucosal lymphatics, the intestines were fixed briefly in $4 \%$ PFA, frozen in Tissue Tek (Sakura) and sectioned. For wholemount visualization of lymphatic mesenteric membrane, the intestines were fixed briefly in 4\% PFA; blocked in $0.5 \%$ BSA, $2 \%$ FBS, and $0.01 \%$ TritonX-100 in PBS; and stained as described above. Antibodies against Proxl (AngioBio), murine Podoplanin (Hybridoma Bank, University of Iowa), cy3-conjugated Sma (Sigma), Cspg4/NG2 (Chemicon), Nrp1, Nrp2, Slc (R\&D Systems), Cd105 (Eng), CD34 (BD Biosciences), and human PDPN (Fitzgerald Industries), were purchased. A guinea pig polyclonal antibody was generated using a fusion protein (kindly provided by Dr. N. Gale) that contained the ecto-hFc region of the mouse Lyvel.

For the whole-mount antibody stainings, pups were immersed overnight in fixative ( $1 \%$ paraformaldehyde in PBS at $\mathrm{pH}$ 7.4). Small intestine, diaphragm and trachea were removed, washed with PBS, and stained immunohistochemically as described previously (Baluk et al. 2007) by incubating whole mounts with primary antibodies against LYVE1 (rabbit polyclonal [Upstate Biotechnologies] or guinea-pig polyclonal [Oliver Laboratory]), Proxl (AngioBio); VE-cadherin (BD Biosciences); PECAM-1/ CD31 (Armenian hamster; Thermo MA); TER119 (rat clone; BD Biosciences), CD41 (Itga2b; eBioscience); Fibrin/fibrinogen (rabbit polyclonal; Dako); PDGFR $\beta$ (rat clone; eBioscience). Secondary antibodies were labeled with FITC, Cy3, or Cy5 (Jackson ImmunoResearch). Specimens were imaged with a Zeiss LSM-510 confocal microscope.

\section{Electron microscopic analysis}

Electron microscopy was performed at the University of Memphis Electron Microscopy Suite. Intestines from P0 Prox $1^{+/-}$ pups and their wild-type littermates underwent whole-mount immunostaining against Lyvel using diaminobenzidine (DAB; Invitrogen) as described previously (Harvey et al. 2005). The tissue was post-fixed in $2.5 \%$ glutaraldehyde in $0.1 \mathrm{M}$ sodium cacodylate buffer for $1 \mathrm{~h}$ and rinsed in three changes of the same buffer. The samples were post-fixed in $2 \%$ osmium tetroxide for $1 \mathrm{~h}$ and dehydrated via a graded series of alcohol, cleared in propylene oxide, embedded in epon araldite, and polymerized overnight at $70^{\circ} \mathrm{C}$. Thick $(0.5-\mu \mathrm{m})$ sections were cut and stained with toluidine blue. Thin (70-nm) sections were cut on a Leica Ultracut E using a diatome diamond knife. The unstained sections were imaged on a JEOL 1200 EX Transmission Electron Microscope with an AMT 2K digital camera.

\section{FITC-dextran}

P5 CAGGCre-ER $R^{T M}$ pups and their respective wild-type littermates were injected by intracardiac perfusion with $8 \mathrm{mg} / \mathrm{mL}$ 
FITC-dextran in 4\%PFA (Sigma) and their intestinal vasculature was immediately visualized under the fluorescence stereomicroscope (Leica).

\section{siRNA analysis}

Primary hLECs (AngioBio) were grown under 5\% $\mathrm{CO}_{2}$ in EGM2 media (Clontech,) containing glutamine, $10 \%$ human off-theclot serum, penicillin, and streptomycin. OnTarget siRNA against human PROX1 (Dharmacon) was introduced into the cell line by using the Cell Transfection System (Amaxa). Scramble siRNA of similar length and relative GC content was used as a negative control. Cells were grown for $72 \mathrm{~h}$ posttransfection, and immunocytochemical analysis was performed by growing cells on eight-chamber slides and then fixing them in $2 \%$ PFA for $10 \mathrm{~min}$ at room temperature and then in $100 \%$ methanol for $1 \mathrm{~min}$ at $0^{\circ} \mathrm{C}$. Cells were washed and immunostained as detailed above. Experiments were performed in triplicate.

\section{Acknowledgments}

We thank Drs. Gopal Murti, Lingqing Zang, and Samuel Connell for their technical expertise and advice with confocal imaging; Dr. Beatriz Sosa-Pineda for advice and helpful discussions; Dr. R. Sathish Srinivasan for valuable suggestions and critical reading of this manuscript; Shelly Self for valuable technical help; Dr. Angela McArthur for excellent editing of this manuscript; members of the Electron Microscopy Cell and Tissue Imaging Center for their help with EM imaging; and Dr. Andy McMahon for the CAGGCre-ER ${ }^{T M}$ strain. This project was supported in part by NIH grants 1F32HD051187-01 (to N.J.), R01-HL073402 (to G.O.), and (HL-24136, HL-59157, and CA-82923) (to D.M.); Cancer Center Support CA-21765; and the American Lebanese Syrian Associated Charities (ALSAC).

\section{References}

Abramsson, A., Lindblom, P., and Betsholtz, C. 2003. Endothelial and nonendothelial sources of PDGF-B regulate pericyte recruitment and influence vascular pattern formation in tumors. J. Clin. Invest. 112: 1142-1151.

Abtahian, F., Guerriero, A., Sebzda, E., Lu, M.M., Zhou, R., Mocsai, A., Myers, E.E., Huang, B., Jackson, D.G., Ferrari, V.A., et al. 2003. Regulation of blood and lymphatic vascular separation by signaling proteins SLP-76 and Syk. Science 299: 247-251.

Backhed, F., Crawford, P.A., O'Donnell, D., and Gordon, J.I. 2007. Postnatal lymphatic partitioning from the blood vasculature in the small intestine requires fasting-induced adipose factor. Proc. Natl. Acad. Sci. 104: 606-611.

Baluk, P., Fuxe, J., Hashizume, H., Romano, T., Lashnits, E., Butz, S., Vestweber, D., Corada, M., Molendini, C., Dejana, E., et al. 2007. Functionally specialized junctions between endothelial cells of lymphatic vessels. J. Exp. Med. 204: 2349-2362.

Breier, G., Breviario, F., Caveda, L., Berthier, R., Schnurch, H., Gotsch, U., Vestweber, D., Risau, W., and Dejana, E. 1996. Molecular cloning and expression of murine vascular endothelial-cadherin in early stage development of cardiovascular system. Blood 87: 630-641.

Breiteneder-Geleff, S., Soleiman, A., Kowalski, H., Horvat, R., Amann, G., Kriehuber, E., Diem, K., Weninger, W., Tschachler, E., Alitalo, K., et al. 1999. Angiosarcomas express mixed endothelial phenotypes of blood and lymphatic capillaries: Podoplanin as a specific marker for lymphatic endothelium. Am. J. Pathol. 154: 385-394.

Cobaleda, C. and Busslinger, M. 2008. Developmental plasticity of lymphocytes. Curr. Opin. Immunol. 2: 139-148.

Cobaleda, C., Jochum, W., and Busslinger, M. 2007. Conversion of mature B cells into T cells by dedifferentiation to uncommitted progenitors. Nature 449: 473-477.

Dudley, A.C., Khan, Z.Z., Shih, S.C., Kang, S.Y., Zwaans, B.M. Bischoff, J., and Klagsbrun, M. 2008. Calcification of multipotent prostate tumor endothelium. Cancer Cell 14: 201211.

Fiedler, U., Christian, S., Koidl, S., Kerjaschki, D., Emmett, M.S., Bates, D.O., Christofori, G., and Augustin, H.G. 2006 The sialomucin CD34 is a marker of lymphatic endothelial cells in human tumors. Am. J. Pathol. 168: 1045-1053.

Foo, S.S., Turner, C.J., Adams, S., Compagni, A., Aubyn, D., Kogata, N., Lindblom, P., Shani, M., Zicha, D., and Adams, R.H. 2006. Ephrin-B2 controls cell motility and adhesion during blood-vessel-wall assembly. Cell 124: 161-173.

Harvey, N.L., Srinivasan, R.S., Dillard, M.E., Johnson, N.C., Witte, M.H., Boyd, K., Sleeman, M.W., and Oliver, G. 2005 Lymphatic vascular defects promoted by Proxl haploinsufficiency cause adult-onset obesity. Nat. Genet. 37: 10721081.

Hayashi, S. and McMahon, A.P. 2002. Efficient recombination in diverse tissues by a tamoxifen-inducible form of Cre: A tool for temporally regulated gene activation/inactivation in the mouse. Dev. Biol. 244: 305-318.

Hong, Y.K., Harvey, N., Noh, Y.H., Schacht, V., Hirakawa, S., Detmar, M., and Oliver, G. 2002. Proxl is a master control gene in the program specifying lymphatic endothelial cell fate. Dev. Dyn. 225: 351-357.

Hong, Y.K., Foreman, K., Shin, J.W., Hirakawa, S., Curry, C.L., Sage, D.R., Libermann, T., Dezube, B.J., Fingeroth, J.D., and Detmar, M. 2004. Lymphatic reprogramming of blood vascular endothelium by Kaposi sarcoma-associated herpesvirus. Nat. Genet. 36: 683-685.

Jones, E.A., le Noble, F., and Eichmann, A. 2006. What determines blood vessel structure? Genetic prespecification vs. hemodynamics. Physiology (Bethesda) 21: 388-395.

Kang, J. and Lee, I. 2006. TuJ1 (class III $\beta$-tubulin) as phenotypic marker of lymphatic and venous valves. Cardiovasc. Pathol. 15: $218-221$.

Karpanen, T., Wirzenius, M., Makinen, T., Veikkola, T., Haisma, H.J., Achen, M.G., Stacker, S.A., Pytowski, B., YlaHerttuala, S., and Alitalo, K. 2006. Lymphangiogenic growth factor responsiveness is modulated by postnatal lymphatic vessel maturation. Am. J. Pathol. 169: 708-718.

Kriehuber, E., Breiteneder-Geleff, S., Groeger, M., Soleiman, A., Schoppmann, S.F., Stingl, G., Kerjaschki, D., and Maurer, D. 2001. Isolation and characterization of dermal lymphatic and blood endothelial cells reveal stable and functionally specialized cell lineages. J. Exp. Med. 194: 797-808.

Lee, Y., Miller, H.L., Jensen, P., Hernan, R., Connelly, M., Wetmore, C., Zindy, F., Roussel, M.F., Curran, T., Gilbertson, R.J., et al. 2003. A molecular fingerprint for medulloblastoma. Cancer Res. 63: 5428-5437.

Lindahl, P., Johansson, B.R., Leveen, P., and Betsholtz, C. 1997. Pericyte loss and microaneurysm formation in PDGF-B-deficient mice. Science 277: 242-245.

Makinen, T., Adams, R.H., Bailey, J., Lu, Q., Ziemiecki, A., Alitalo, K., Klein, R., and Wilkinson, G.A. 2005. PDZ interaction site in ephrinB2 is required for the remodeling of lymphatic vasculature. Genes \& Dev. 19: 397-410.

Makinen, T., Norrmen, C., and Petrova, T.V. 2007. Molecular mechanisms of lymphatic vascular development. Cell. Mol. 
Life Sci. 64: 1915-1929.

Oliver, G. and Detmar, M. 2002. The rediscovery of the lymphatic system: Old and new insights into the development and biological function of the lymphatic vasculature. Genes \& Dev. 16: 773-783.

Oliver, G. and Srinivasan, R.S. 2008. Lymphatic vasculature development: Current concepts. Ann. N. Y. Acad. Sci. 1131: 75-81.

Ozerdem, U., Monosov, E., and Stallcup, W.B. 2002. NG2 proteoglycan expression by pericytes in pathological microvasculature. Microvasc. Res. 63: 129-134.

Petrova, T.V., Makinen, T., Makela, T.P., Saarela, J., Virtanen, I., Ferrell, R.E., Finegold, D.N., Kerjaschki, D., Yla-Hertualla, S., and Alitalo, K. 2002. Lymphatic endothelial reprogramming of vascular endothelial cells by the Prox-1 homeobox transcription factor. EMBO J. 21: 4593-4599.

Petrova, T.V., Karpanen, T., Norrmen, C., Mellor, R., Tamakoshi, T., Finegold, D., Ferrell, R., Kerjaschki, D., Mortimer, P., Yla-Herttuala, S., et al. 2004. Defective valves and abnormal mural cell recruitment underlie lymphatic vascular failure in lymphedema distichiasis. Nat. Med. 10: 974-981.

Roca, C. and Adams, R.H. 2007. Regulation of vascular morphogenesis by Notch signaling. Genes \& Dev. 21: 2511-2524.

Sabin, F. 1902. On the origin of the lymphatics system from the veins and the development of the lymph hearts and the thoracic duct in the pig. Am. J. Anat. 1: 367-391.

Schacht, V., Ramirez, M.I., Hong, Y.K., Hirakawa, S., Feng, D., Harvey, N., Williams, M., Dvorak, A.M., Dvorak, H.F., Oliver, G., et al. 2003. T1 $\alpha$ /podoplanin deficiency disrupts normal lymphatic vasculature formation and causes lymphedema. EMBO J. 22: 3546-3556.

Takahashi, K., Tanabe, K., Ohnuki, M., Narita, M., Ichisaka, T., Tomoda, K., and Yamanaka, S. 2007. Induction of pluripotent stem cells from adult human fibroblasts by defined factors. Cell 131: 861-872.

Verfaillie, C.M. 2008. Bony endothelium: Tumor-mediated transdifferentiation? Cancer Cell 14: 193-194.

Wang, H.W., Trotter, M.W., Lagos, D., Bourboulia, D., Henderson, S., Makinen, T., Elliman, S., Flanagan, A.M., Alitalo, K., and Boshoff, C. 2004. Kaposi sarcoma herpesvirus-induced cellular reprogramming contributes to lymphatic endothelial gene expression in Kaposi sarcoma. Nat. Genet. 36: 687693.

Wigle, J.T. and Oliver, G. 1999. Prox1 function is required for the development of the murine lymphatic system. Cell 98: 769-778.

Wigle, J.T., Harvey, M., Detmar, M., Lagutina, I., Grosveld, G., Gunn, M.D., Jackson, D.G., and Oliver, G. 2002. An essential role for Proxl in the induction of the lymphatic endothelial cell phenotype. EMBO J. 21: 1505-1513.

Wikstrom, P., Lissbrant, I.F., Stattin, P., Egevad, L., and Bergh, A. 2002. Endoglin (CD105) is expressed on immature blood vessels and is a marker for survival in prostate cancer. Prostate 51: 268-275.

Xie, H., Ye, M., Feng, R., and Graf, T. 2004. Stepwise reprogramming of B cells into macrophages. Cell 117: 663-676.

You, L.R., Lin, F.J., Lee, C.T., DeMayo, F.J., Tsai, M.J., and Tsai, S.Y. 2005. Suppression of Notch signalling by the COUPTFII transcription factor regulates vein identity. Nature 435: 98-104.

Zaret, K.S. 2008. Genetic programming of liver and pancreas progenitors: Lessons for stem-cell differentiation. Nat. Rev. Genet. 9: 329-340. 


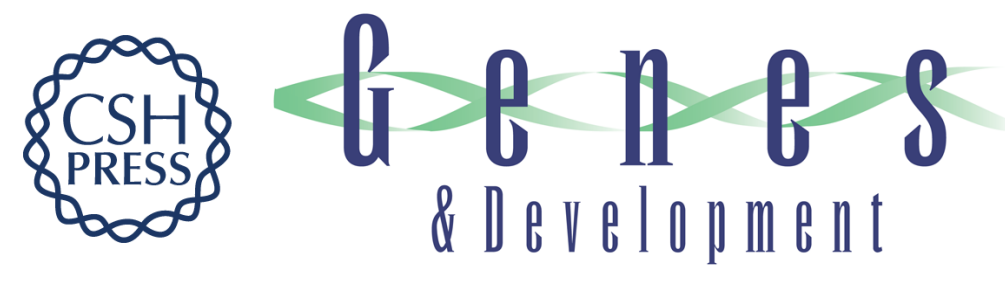

\section{Lymphatic endothelial cell identity is reversible and its maintenance requires Prox1 activity}

Nicole C. Johnson, Miriam E. Dillard, Peter Baluk, et al.

Genes Dev. 2008, 22:

Access the most recent version at doi:10.1101/gad.1727208

\section{Supplemental http://genesdev.cshlp.org/content/suppl/2008/11/18/22.23.3282.DC1 \\ Material}

Related Content Master and commander: continued expression of Prox1 prevents the dedifferentiation of lymphatic endothelial cells

M. Gabriele Bixel and Ralf $\mathrm{H}$. Adams

Genes Dev. December , 2008 22: 3232-3235

References This article cites 40 articles, 13 of which can be accessed free at:

http://genesdev.cshlp.org/content/22/23/3282.full.html\#ref-list-1

Articles cited in:

http://genesdev.cshlp.org/content/22/23/3282.full.html\#related-urls

\section{License}

Email Alerting

Service

Receive free email alerts when new articles cite this article - sign up in the box at the top right corner of the article or click here.

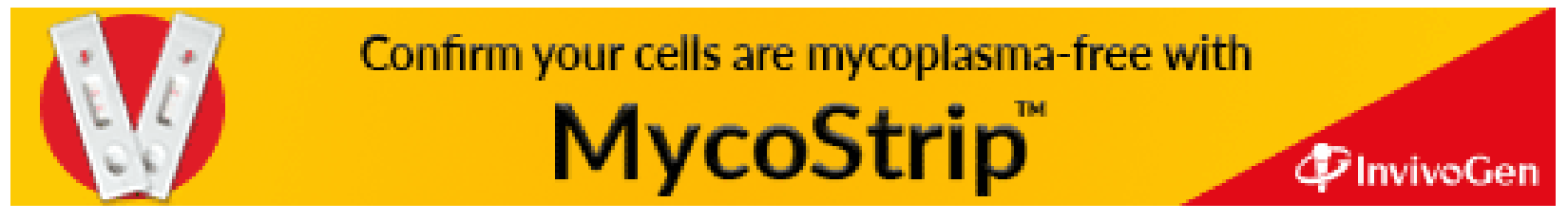

